\title{
Surfactant protein B deficiency caused by homozygous mutation in a term neonate
}

Deniz Yılmaz, ${ }^{1}$ Firat Ozkececi, ${ }^{2}$ Turan Derme, ${ }^{2}$ Belma Saygili Karagol

University of Health Sciences, Gulhane Medical Faculty, Department of Pediatrics, Ankara, Turkey

University of Health Sciences, Gulhane Medical Faculty, Department of Pediatrics, Division of

Veonatology, Ankara, Turkey

Introduction: Surfactant protein B (SP-B) deficiency is a rare autosomal recessive disorder that typically presents in neonates early after birth with respiratory distress and the need for mechanical ventilation, mimicking hyaline membrane disease in the preterm infant. Initial radiographs show lung opacification, and the disorder may transiently respond to exogenous surfactant therapy. A fatal outcome is the norm despite supportive respiratory strategies unless lung transplantation is performed in the first months. Here we report on a case of fatal Surfactant Protein B Deficiency presenting with respiratory distress on the first day of life in a term baby.

Case: A $3710 \mathrm{~g}$ male infant was born by normal vaginal delivery at 38 weeks to a 38-year-old gravid 3, para 2 mother with consanguineous marriage. Apgar scores were 7/10/10 at $1 / 5 / 10$ minutes. At the age of 3 hours, the infant was transferred to the neonatal intensive care unit presenting with tachypnea (RR76/min), pale skin, expiratory wheezing and respiratory distress. Laboratory findings revealed respiratory acidosis (PCO2:64mmHg). Chest X-ray showed abnormal radiography findings as bilateral ground glass with positive air bronchogram. Mechanical ventilation was initiated becaouse of worsened respiratory status and Surfactant replacement therapy resulted in acute improvement of oxygenation. Congenital heart disease was excluded by echocardiography. High-frequency oscillatory ventilation (HFOV), as well as repeated surfactant supplementation and subsequent conventional mechanical ventilation resulted in solely intermittant respiratory improvement and oxygen demand persisted in following days. As far as surfactant dysfunction disorder was suspected., SP gene analysis from a blood sample was performed. Genetic analysis revealed SP-B deficiency caused by a homozygous mutation on exon 6 and 7 of the SP-B gene. At the age of 39 days, the baby died due to respiratory failure.
Result: Surfactant protein B (SP-B) deficiency is a rapidly fatal disorder that typically presents in neonates ealy after birth with respiratory distress and the need for mechanical ventilation. A search for SP-B mutations should be included in the assessment of neonates with severe diffuse pulmonary disease and rapidly progressive disease.Gene therapy is hoped to be an option in this fatal disorder in the future.

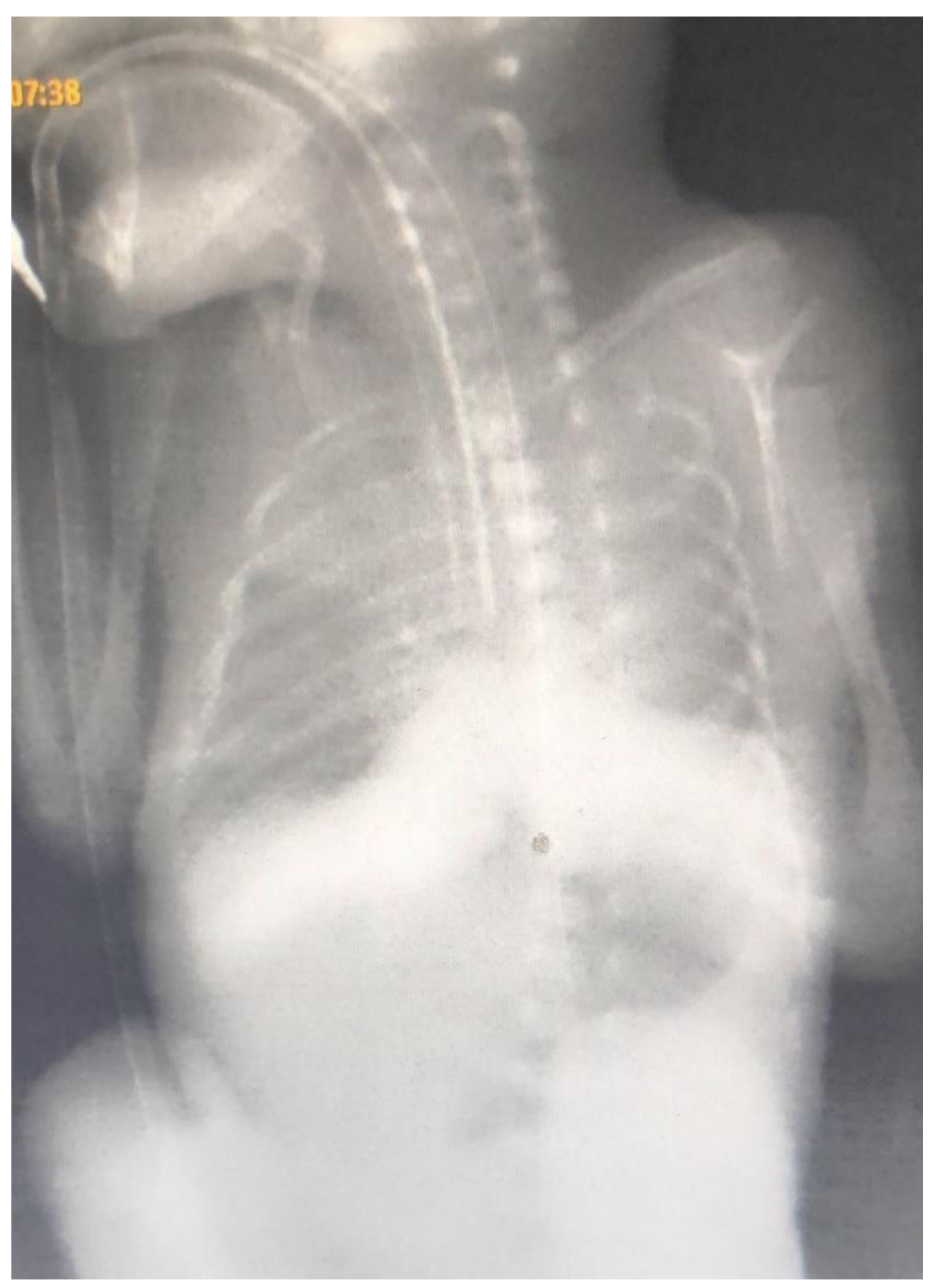

